\title{
Epidemiological perspectives of ticks and tick-borne diseases in South Sudan: Cross-sectional survey results
}

\author{
Authors: \\ Fredrick M. Kivaria ${ }^{1}$ \\ Angolwisye M. Kapaga ${ }^{2}$ \\ Gabriel K. Mbassa ${ }^{3}$ \\ Paul F. Mtui ${ }^{4}$ \\ Rhombe J. Wani ${ }^{5}$ \\ Affiliations: \\ ${ }^{1}$ National Epidemiology \\ Section, Ministry of Livestock \\ and Fisheries Development, \\ Tanzania \\ ${ }^{2}$ Central Veterinary \\ Laboratory, Dar es Salaam, \\ Tanzania \\ ${ }^{3}$ Faculty of Veterinary \\ Medicine, Sokoine University \\ of Agriculture, Tanzania \\ ${ }^{4}$ Veterinary Investigation \\ Centre, Arusha, Tanzania \\ ${ }^{5}$ Ministry of Animal \\ Resources and Fisheries, \\ Government of South Sudan, \\ South Sudan

\section{Correspondence to:} \\ Fredrick Kivaria \\ Email: \\ fredkiv@gmail.com \\ Postal address: \\ PO Box 9152, Dar es Salaam, \\ Tanzania \\ Dates: \\ Received: 09 Jan. 2012 \\ Accepted: 11 May 2012 \\ Published: 03 Sept. 2012 \\ How to cite this article: \\ Kivaria, F.M., Kapaga, \\ A.M., Mbassa, G.K., Mtui, \\ P.F. \& Wani, R.J., 2012, \\ 'Epidemiological perspectives \\ of ticks and tick-borne \\ diseases in South Sudan: \\ Cross-sectional survey \\ results', Onderstepoort \\ Journal of Veterinary \\ Research 79(1), Art. \#400, \\ 10 pages. $\mathrm{http}: / / \mathrm{dx}$.doi. \\ org/10.4102/ojvr.v79i1.400
}

(C) 2012. The Authors. Licensee: AOSIS OpenJournals. This work is licensed under the Creative Commons Attribution License.
A cross-sectional study was conducted between September and October 2010 in five states of South Sudan that were selected on the basis of the perceived risk of tick-borne diseases. The purpose was to investigate epidemiological parameters of tick-borne diseases in South Sudan and their uses in future control strategies. A total of 805 calves were assessed by clinical, microscopic and serological examination and tick counts. The indirect Enzyme-Linked Immuno-Sorbent Assay (ELISA) was used to detect antibodies to Theileria parva, Theileria mutans, Anaplasma marginale and Babesian bigemina. Sero-conversion risks for T. parva and T. mutans were $27.3 \%$ and $31.3 \%$ respectively, whilst the risk was $57.6 \%$ and $52.8 \%$ for A. marginale and B. bigemina, respectively. Major tick species identified include Rhipicephalus appendiculatus, Rhipicephalus decoloratus, Rhipicephalus microplus, Amblyomma variegatum, and Rhipicephalus evertsi. There was great variation $(P \leq 0.001)$ in the number of all these ticks, both between herds in a state and between calves in an individual herd. The low and intermediate sero-conversion risks observed in the study states suggest that immunisation against East Coast fever (ECF) is justified. Fortunately, three major genotypes that were identified by applying Polymerase Chain Reaction Restriction Fragment Length Polymorphism (PCRRFLP) analysis on the p104 to the blood samples and T. parva Muguga, matched very well with T. parva Kiambu 5 and T. parva Muguga; therefore the Muguga cocktail can be used for the immunisation of cattle in South Sudan. However, prospective studies are required to develop optimal control measures for tick-borne diseases under different ecological and husbandry practices in South Sudan.

\section{Introduction}

Infection with Theileria parva causes the disease, East Coast fever (ECF), also known as bovine theileriosis. The disease is endemic to the region stretching from the Democratic Republic of the Congo to Tanzania, from South Sudan to South Africa; with a heterogeneous distribution and prevalence strongly related to its vector dynamics, host susceptibility, grazing system and tickcontrol practices (Kivaria 2007; Marufu et al. 2010; Rubaire-Akiiki et al. 2006). In all affected areas, ECF has a considerable epidemiological and economic significance; in Tanzania; the disease is the main cause of reported cattle deaths and was estimated to account for $68 \%$ of the 364 million USD annual total losses resulting from tick-borne diseases (Kivaria 2006). In unvaccinated zebu calves raised by pastoralists, ECF is responsible for an annual mortality risk of $40 \%-80 \%$ (Kivaria 2006). The first outbreak of bovine theileriosis in Sudan was reported in 1950 (Hoogstraal 1956). After the 1950-outbreak, the disease was not reported again until 1981. Since then many outbreaks of ECF have been reported in Southern Sudan (Julla, Tingwa \& Kwajok 1989; Julla 1993). In 1983, an outbreak was reported in Palotaka, Nimule and Juba Township with calf mortalities estimated at $80 \%-100 \%$ (Julla 1985).

Unpublished archives indicate that the disease has been restricted previously to the Equatorial Region of South Sudan, probably because of its proximity to ECF-infected zones in Kenya, Uganda, and north-east Congo, coupled with the suitable climatic conditions for the survival of the vector. During the two decades of civil war in South Sudan, pastoralists from Jonglei State were displaced and moved southward with their cattle to ECF-infected areas in Central Equatoria State. The gradual introduction of these naïve animals into the infected zone generated some degree of immunity against the disease and the animals were able to survive the tick and parasite challenge. After the Comprehensive Peace Agreement (CPA) in 2005, Dinka Bor of Jonglei State started moving northward with huge numbers of livestock. In June 2008, the first outbreak of ECF was reported in an area called Lek Yek in Bor town, and thereafter, a cycle of outbreaks followed and continued throughout 2009 and 2010, affecting animal production and health as well as the food security of many households whose livelihoods are dependent mainly on livestock.

Results of several tick-borne diseases (TBD) studies in smallholder dairy and pastoral livestock farming systems in Eastern Africa indicated that TBDs' sero-prevalence risk differs across agro- 
ecological zones and grazing systems (Bazarusanga et al. 2007; Maloo et al. 2001; Rubaire-Akiiki et al. 2004; Swai et al. 2005). The impetus to carry out this cross-sectional study was borne out of the desire to characterise the epidemiological parameters (sero-conversion risk, morbidity and mortality risks) and to document the dynamics of tick infestation of cattle and the infection patterns of bovine theileriosis, babesiosis and anaplasmosis, because these are very important factors to consider when planning production system-specific control strategies of TBD. In this paper we use these factors to characterise the epidemiological states of ECF and other tick-borne diseases, namely anaplasmosis and babesiosis in Central Equatoria (CE), Eastern Equatoria (EE), Jonglei (JNG), Lakes (LKS) and Western Equatoria (WE) States of South Sudan. In addition, molecular characterisation of T. parva isolates was performed, and this information was combined to assess the current and potential TBD status in each state as well as the implications for future control strategies.

\section{Materials and methods \\ Sample size}

This cross-sectional study was designed and implemented between September and October 2010. One methodological challenge faced during the execution of this survey was the lack of information on husbandry practices and estimated herd level prevalence of tick-borne diseases. Deem et al. (1993) suggested that a sample size of 10-20 pastoral herds, that contained an average of 10 calves per herd, would be adequate across a range of epidemiological states, with sample sizes at the top of the range required in epidemiologically unstable areas. Based on these results, it was decided to select 20 cattle camps randomly from each state and six calves from each selected camp. A three-stage sampling was employed. Payams or divisions were purposefully selected first, based on the perceived risk of tick-borne disease. It was followed by a random selection of cattle camps or boma, and then animals were randomly selected from each cattle camp. The resulting final sample size therefore depended on the number of camps and calves sampled from each state and cattle camps, respectively. The geographical location of the five states, counties and the cattle camps investigated during the study period, have been depicted (Figure 1).

The target age group was calves of age 4-18 months. Aging was performed based on the herdsman-derived information and on the absence of permanent incisors. All herdsmen were questioned on the occurrence of tick-borne diseases, herd size, calf mortality, descriptions of clinical signs observed in calves that had died, acaricide usage, treatment, distance travelled to water and grazing, and the general descriptions of tick problems present in each herd.

\section{Clinical and laboratory investigation}

Each animal was subjected to clinical examination, including the measurement of rectal temperature. A temperature $\geq 39.5^{\circ} \mathrm{C}$ in adults (>12 months) and a temperature $\geq 40{ }^{\circ} \mathrm{C}$ in calves ( $\leq 12$ months) were considered a febrile reaction. With regard to the palpation of the pre-scapula lymph nodes, the sizes were scored as + (normal); ++ (slightly enlarged) and +++ (grossly enlarged). In this study, lymph node enlargement of $(+)$ were defined as originating from animals free of theileriosis, whilst palpation results of $\geq++$ were classified as evidence of theileriosis. Blood smears and lymph node biopsies were taken to demonstrate the presence of the causing organism. Smears were stained with Giemsa and microscopically examined under $40 \mathrm{x}$ and $100 \mathrm{x}$ oil immersion objectives.

\section{Tick counts and tick infection proportions}

Tick infestation was estimated by counting the number of adult ticks (male, female, engorged and non-engorged) on the body of each calf examined. Ticks are not randomly distributed on the animal body but rather restricted to a few predilection sites; therefore we preferred to count all the ticks on one side of the animal body instead of making counts per square meter. The ticks on one side of the body of each calf were counted and categorised into species; visual identification was carried out during the tick counts, based on the guidelines described by Walker et al. (2003).

Adult Rhipicephalus appendiculatus that were partially engorged and thus potentially with developing sporoblast stages of $T$. parva, were dissected to remove the salivary glands for examination of infection with T. parva, as described by Buscher and Otim (1986). Tick infections were determined through direct microscopy after staining the tick salivary glands with Schiff's (Fuelgen's) reagent to identify infected acini. The intensity of infection was subsequently estimated by counting the number of infected acini.

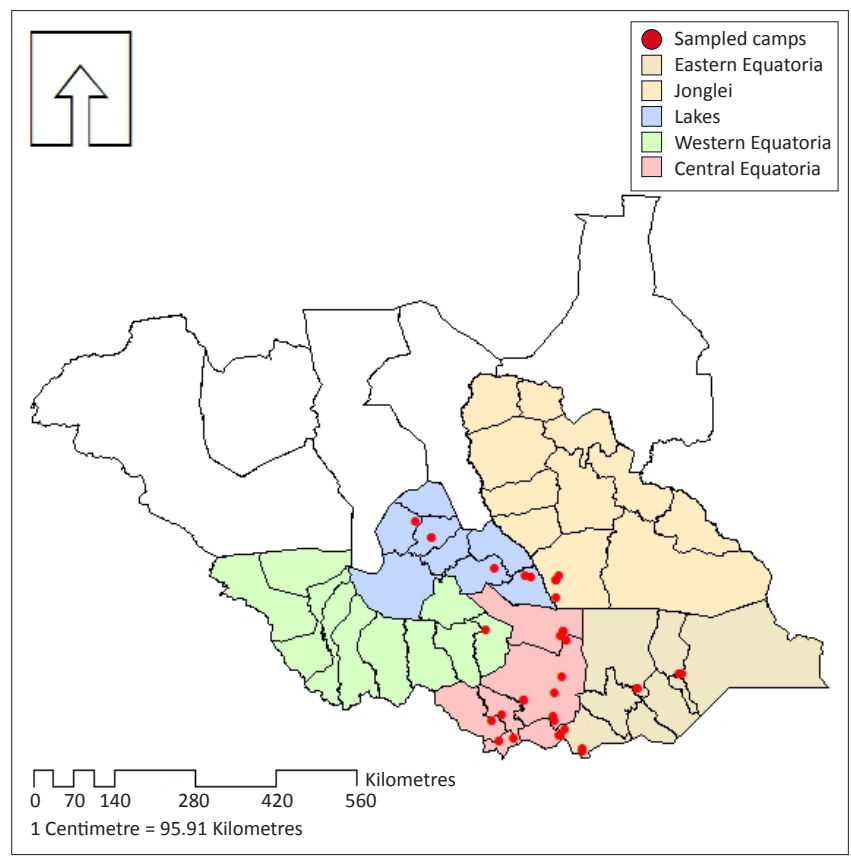

FIGURE 1: Geographical location of the five states, counties and the cattle camps investigated during the cross-sectional survey (September 2010 - October 2010). 


\section{Serological tests}

Blood was collected from each calf in one $10-\mathrm{mL}$ plain Vacutainer tube (Becton Dickinson Vacutainer Systems, UK) by jugular venepuncture. Tubes were labelled and verified before drawing the blood from the calves. After collection, blood samples were stored in a car refrigerator until sera could be separated (usually within 24 hours). The sera were separated by centrifugation at $3000 \mathrm{xg}$ for $20 \mathrm{~min}$ and divided into aliquots and stored in freezers $\left(-20^{\circ} \mathrm{C}\right)$ in the laboratory.

Enzyme-Linked Immuno-Sorbent Assays (ELISAs) were used to detect antibodies to T. parva, Theileria mutans, Anaplasma marginale and Babesian bigemina. Polymorphic immune-dominant molecule (PIM) recombinant antigen was used as described by Katende et al. (1998) for the detection of T. parva antibodies. Tests for antibodies to T. mutans and B. bigemina have been described by Katende et al. $(1990,1998)$. Each ELISA test plate included predetermined positive and negative control sera. Optical density (OD) readings from the reference highly positive control sera were used to compute the per cent positivity (PP) for the test sera (Wright et al. 1993). Per cent positivity for test serum was expressed as the per cent of the test serum OD (at optimum dilution of 1:200) divided by the mean OD reading derived from the strong positive control serum on the linear curve (from a curve of OD against the reciprocal of serial dilutions) (Katende et al. 1998; Wright et al. 1993). For T. parva and T. mutans, a sample was considered positive if the PP value equalled 20 or higher, whilst the cut-off point was 15 for A. marginale and B. bigemina. The risk of exposure of animals to T. parva was estimated by the sero-conversion risk. The sero-conversion risk was computed as the number of animals sero-converted divided by the total number of animals tested. Tick identification, microscopic and serological work were conducted at the Veterinary Investigation Centre in Arusha, Tanzania.

\section{Characterisation of epidemiological states of tick-borne diseases}

The epidemiological state of tick-borne diseases in an area can be considered as either endemically stable or unstable. For babesiosis and anaplasmosis, antibody prevalence has been used as an indicator of the existence of endemic stability, and thus the biological evidence of the need for vaccination or for no intervention (De Vos 1992; Mahoney 1977). In broad terms, there is a strong correlation between high antibody prevalence to these infections and endemic stability, and low antibody prevalence and endemic instability. This phenomenon also exists with T. parva, but it is by no means absolute and it is complicated by various factors. Endemic stability is assessed based on a combination of indicators (Norval, Perry \& Young 1992) including (1) antibody prevalence, (2) disease incidence, (3) case-fatality proportion and (4) age group affected (Norval et al. 1992; Perry \& Young 1995). Tick-infection proportions are also considered helpful (Perry et al. 1992). According to Norval et al. (1992), an endemically stable state is characterised by high antibody prevalence, low disease incidence and case- fatality proportion, and a rapid acquisition of infection in young calves. An endemically unstable state is characterised by the opposite, that is, lower antibody prevalence, high disease incidence and case-fatality proportion and primary infections that occur in all age groups. These criteria were used to characterise the endemic stability of the five states investigated.

\section{Molecular characterisation of Theileria parva isolates from South Sudan}

Lymph node biopsy and blood samples collected from cattle were used as the source of deoxyribonucleic acid (DNA) for Polymerase Chain Reaction (PCR) analysis. Genomic DNA was extracted from blood samples received from Southern Sudan. Deoxyribonucleic acid was also extracted from a stabilate of T. parva Muguga (Muguga, Kiambu 5, Serengeti transformed) obtained from the Ministry of Livestock and Fisheries Development, Dar es Salaam, and used as controlDNA for the processes in the characterisation. The DNA extraction and PCR assays were conducted as described by Bishop (2001).

\section{Ethical considerations}

Field observations were conducted and samples collected from animals. No experiments were performed on the animals and the animals used in the collection of samples for the study were treated humanely.

\section{Results}

A total of 822 calves from the 5 states, 16 counties, 29 Payams and 34 cattle camps were investigated; 805 calves had complete observations, whilst 17 calves did not provide all the information, and were therefore omitted from further analyses. Of the 805 calves investigated, $50.4 \%$ were female calves, whilst $49.6 \%$ were male calves; the mean age for female calves was 9.34 months and for male calves 8.98 months. The mean age of the studied calves was 9.16 months, whilst minimum and maximum ages were 0.75 months and 24.00 months, respectively.

\section{Temperature, lymph node scores, biopsies and blood smears}

The average temperature was $38.0^{\circ} \mathrm{C} \pm 0.16_{\mathrm{SE}^{\prime}}$ the median was $38.20^{\circ} \mathrm{C}$ and the mode was $41^{\circ} \mathrm{C}$; there was no significant $\left(X^{2}=6.342\right)$ statistical difference in the distribution of temperature recordings amongst the states.

A total of 805 pre-scapula lymph nodes were examined, of which $45.0 \%$ were positive, with reactions of ++ or higher; $55.0 \%$ of the 805 pre-scapula lymph nodes showed a negative reaction of + . Palpation results of ++ and +++ accounted for $34.6 \%$ and $10.4 \%$, of the palpated lymph nodes respectively. Of the 362 grossly enlarged pre-scapula lymph nodes, 3.0\% (11) were positive for schizonts; the remaining smears were negative. Theileria parva piroplasms were detected in 32 corresponding blood smears, and 6 out of the 32 T. parva positive blood smears were a mixed infection between T. parva and $A$. marginale. 
TABLE 1: The distribution of the haemo-parasites detected in five states of South Sudan during a cross-sectional survey (September 2010 - October 2010).

\begin{tabular}{|c|c|c|c|c|c|}
\hline \multirow[t]{2}{*}{ State } & \multicolumn{5}{|c|}{ Parasites seen } \\
\hline & Theileria parva & Anaplasma marginale & $\begin{array}{l}\text { Theileria parva and } \\
\text { Anaplasma marginale }\end{array}$ & $\begin{array}{l}\text { Babesia bigemina and } \\
\text { Anaplasma marginale }\end{array}$ & Trypanosome species \\
\hline Central Equatoria & 8 & 1 & 1 & 0 & 0 \\
\hline Jonglei & 6 & 9 & 2 & 0 & 1 \\
\hline Lakes & 7 & 12 & 2 & 0 & 0 \\
\hline West Equatoria & 0 & 1 & 0 & 0 & 0 \\
\hline
\end{tabular}

TABLE 2: Variation in the total number of ticks and the individual tick species on calves in the study states, during the cross-sectional survey (September 2010 - October 2010)

\begin{tabular}{|c|c|c|c|c|c|}
\hline \multirow[t]{2}{*}{ Ticks } & \multirow[t]{2}{*}{ State } & \multicolumn{3}{|c|}{ Tick counts } & \multirow[t]{2}{*}{$P$} \\
\hline & & Minimum & Maximum & Median & \\
\hline \multirow[t]{5}{*}{ Rhipicephalus appendiculatus } & Central Equatoria & 0 & 480 & 0 & $\leq 0.001$ \\
\hline & Eastern Equatoria & 0 & 832 & 0 & \\
\hline & Jonglei & 0 & 240 & 10 & \\
\hline & Lakes & 0 & 240 & 6 & \\
\hline & Total & - & 1792 & - & \\
\hline \multirow[t]{5}{*}{ Rhipicephalus decoloratus } & Central Equatoria & 0 & 384 & 0 & $\leq 0.001$ \\
\hline & Eastern Equatoria & 0 & 112 & 0 & \\
\hline & Jonglei & 0 & 368 & 0 & \\
\hline & Lakes & 0 & 192 & 12 & \\
\hline & Total & - & 1056 & - & \\
\hline \multirow[t]{5}{*}{ Amblyomma variegatum } & Central Equatoria & 0 & 736 & 0 & $\leq 0.001$ \\
\hline & Eastern Equatoria & 0 & 544 & 0 & \\
\hline & Jonglei & 0 & 128 & 9 & \\
\hline & Lakes & 0 & 352 & 0 & \\
\hline & Total & - & 1760 & - & \\
\hline \multirow[t]{5}{*}{ Rhipicephalus evertsi evertsi } & Central Equatoria & 0 & 256 & 0 & $\leq 0.001$ \\
\hline & Eastern Equatoria & 0 & 336 & 0 & \\
\hline & Jonglei & 0 & 32 & 0 & \\
\hline & Lakes & 0 & 256 & 0 & \\
\hline & Total & - & 880 & - & \\
\hline \multirow[t]{5}{*}{ Hyalomma truncatum } & Central Equatoria & 0 & 0 & 0 & $\leq 0.001$ \\
\hline & Eastern Equatoria & 0 & 32 & 0 & \\
\hline & Jonglei & 0 & 64 & 11 & \\
\hline & Lakes & 0 & 224 & 0 & \\
\hline & Total & - & 320 & - & \\
\hline Rhipicephalus microplus & Lakes & 0 & 32 & 7 & \\
\hline
\end{tabular}

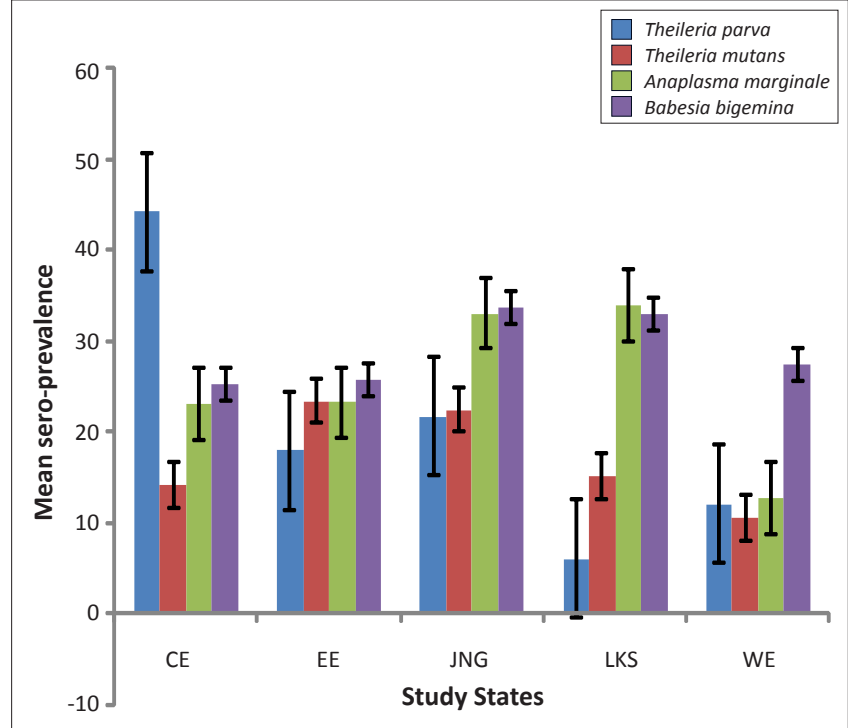

CE, Central Equatoria State; EE, Eastern Equatoria State; JNG, Jonglei State; LKS, Lakes State; WE, Western Equatoria State.

FIGURE 2: Mean serum antibody sero-prevalence of 805 calves investigated during the cross-sectional survey (September 2010 - October 2010).
Blood parasites were detected in $8.9 \%$ (72) of the 805 blood smears examined; the haemo-parasites detected include T. parva (26), A. marginale (38), T. parva and A. marginale (6), $B$. bigemina and $A$. marginale (1), and trypanosome species (1). Because of the small number of haemo-parasites detected, no statistical comparisons were made for the distribution of these parasites by states; the distribution of these haemoparasites by States has been summarised (Table 1).

\section{Theileria parva, Theileria mutans, Anaplasm marginale and Babesia bigemina antibody patterns}

Serological results indicated that $27.3 \%$ (220) of the 805 calves screened have sero-converted against T. parva, and $72.7 \%$ (585) were negative, whilst for T. mutans 31.3\% (252) and $68.7 \%$ (553) of the 805 calves screened were sero-positive and negative, respectively. On the other hand, 57.6\% (464) and $52.8 \%$ (425) of the 805 calves were sero-positive for A. marginale and B. bigemina antibodies, respectively. The mean antibody sero-prevalence with standard errors for T. parva, T. mutans, A. marginale and B. bigemina by states are 
shown (Figure 2). Central Equatoria State was significantly associated with higher sero-prevalence compared with other states.

The relationship between the calf age and sero-prevalence (Figure $3 a-d)$ shows that the majortiy of the calves are born with very little (negative sign) maternal antibodies to the major tick-borne parasites studied. They sero-convert, however, by the age of 3 months, the age at which, according to the herdsmen, calves go out for grazing. However, the acquired antibodies quickly decay with advancing age, in such a way that the majority of the calves have less than $15 \%$ sero-prevalence at 11 months of age.

\section{Tick infestation patterns}

The occurrence and distribution of ticks in the study states have been listed (Table 2). The highest number of tick counts was made in the Eastern Equatoria and Central Equatoria States. The most prevalent ticks were $R$. appendiculatus and Amblyoma variegatum and these were observed in Eastern Equatoria and Central Equatoria States, respectively. It is further shown (Table 2) that infestation by ticks (total ticks and individual species) significantly $(P \leq 0.001)$ varied with states, especially in Eastern Equatoria and Central Equatoria States; there was also great variation in the number of ticks between calves in an individual herd. No ticks were collected from the Western Equatoria State. The numbers of $R$. appendiculatus dissected, the proportion infected with T. parva and the intensity of those infections, have been listed (Table 3). The prevalence of infections in dissected ticks was in the range $25 \%-28 \%$.

\section{Epidemiological states of Theileria parva, Theileria mutans, Anaplasma marginale and Babesia bigemina infections}

Enzyme-Linked Immuno-Sorbent Assays results for T. mutans and B. bigemina have been summarised (Table 4) and these results were used to classify the five states for endemic stability. Tabulated values show that all states are endemically unstable to T. mutans, but moderately stable to $B$. bigemina. No morbidity and mortality risk data for T. mutans and B. bigemina were available. The estimates of indicators used to classify the five states for endemic stability to ECF (Table 5) and anaplasmosis (Table 6) have been listed. All strata were judged to be endemically unstable; however, the degree of their instability varied. We assessed the relative stability of each state over the range of endemic stability or instability (Tables 5 and 6).

\section{Molecular characterisation of Theileria parva}

The profiles generated by applying Polymerase Chain Reaction Restriction Fragment Length Polymorphism (PCRRFLP) analysis on the p104 to the samples and T. parva Muguga were obtained (Table 7). Three major genotypes were identified. The first genotype appeared exactly the same as T. parva Muguga and T. parva Kiambu 5 at p104 locus with
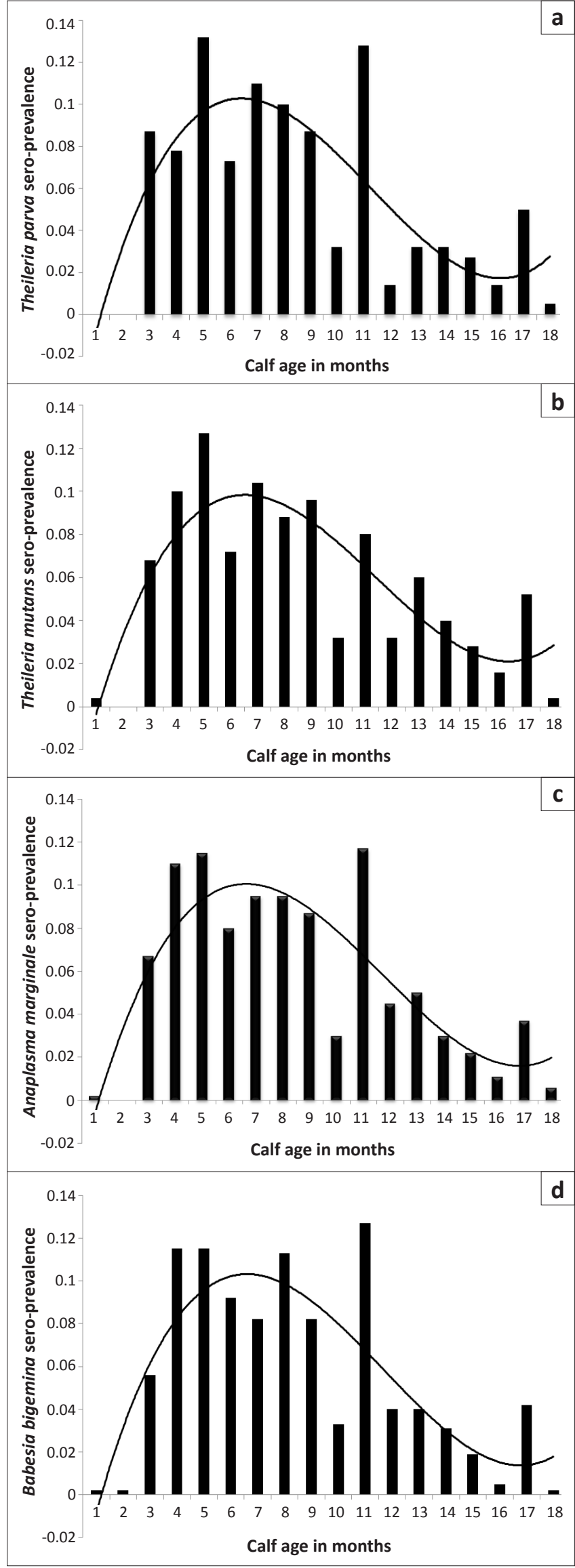

FIGURE 3: Relationship between sero-prevalence for (a) Theileria parva, (b) Theileria mutans, (c) Anaplasma marginale and (d) Babesia bigemina, and calf age (in months) from five states of South Sudan (September 2010 - October 2010). 
TABLE 3: Prevalence and intensity of Theileria parva infections in non-engorged adult Rhipicephalus appendiculatus collected from calves in five states of South Sudan (September 2010 - October 2010).

\begin{tabular}{|c|c|c|c|c|c|c|}
\hline \multirow[t]{2}{*}{ State } & \multirow[t]{2}{*}{ Ticks collected } & \multirow[t]{2}{*}{ Number dissected } & \multicolumn{2}{|c|}{ Number positive for Theileria parva infection } & \multirow[t]{2}{*}{ Total ticks infected } & \multirow[t]{2}{*}{ Total mean intensity: } \\
\hline & & & $N$ & $\%$ & & \\
\hline Central Equatoria & 480 & 52 & $13 \dagger$ & 25 & 93 & 7.15 \\
\hline Eastern Equatoria & 832 & 80 & 20 & 25 & 121 & 6.05 \\
\hline Jonglei & 240 & 36 & 10 & 28 & 83 & 8.30 \\
\hline Lakes & 240 & 36 & 9 & 25 & 84 & 9.33 \\
\hline Total & 1792 & 204 & 52 & 25 & 3381 & 7.33 \\
\hline
\end{tabular}

$N$, used as means of number.

$\uparrow$, Computed as number positive for Theileria parva infection or number dissected.

$\$$, Computed as total number of ticks infected or number positive for Theileria parva infection.

TABLE 4: Distribution of Theileria mutans and Babesia bigemina sero-prevalence by states.

\begin{tabular}{|c|c|c|c|c|c|c|c|c|}
\hline \multirow[t]{4}{*}{ State } & \multicolumn{8}{|c|}{ Parasites } \\
\hline & \multicolumn{4}{|c|}{ Theilaria mutans } & \multicolumn{4}{|c|}{ Babesia bigemina } \\
\hline & \multicolumn{2}{|c|}{ Negative } & \multicolumn{2}{|c|}{ Positive } & \multicolumn{2}{|c|}{ Negative } & \multicolumn{2}{|c|}{ Positive } \\
\hline & $N$ & $\%$ & $N$ & $\%$ & $N$ & $\%$ & $N$ & $\%$ \\
\hline Central Equatoria & 144 & 78.30 & 40 & 21.70 & 93 & 50.50 & 91 & 49.50 \\
\hline Western Equatoria & 12 & 75.00 & 4 & 25.00 & 8 & 50.00 & 8 & 50.00 \\
\hline Eastern Equatoria & 116 & 58.00 & 84 & 42.00 & 117 & 58.50 & 83 & 41.50 \\
\hline Jonglei & 125 & 62.50 & 75 & 37.50 & 88 & 44.00 & 112 & 56.00 \\
\hline Lakes & 154 & 75.90 & 49 & 24.10 & 74 & 36.50 & 129 & 63.50 \\
\hline
\end{tabular}

$N$, used as a means of number.

TABLE 5: Criteria used to characterise endemic stability to bovine theileriosis in the five states studied in South Sudan.

\begin{tabular}{|c|c|c|c|c|c|c|}
\hline States & $\begin{array}{c}\text { Theileria parva } \\
\text { sero-prevalence } \dagger(\%)\end{array}$ & $\begin{array}{c}\text { Theileria } \\
\text { morbidity: }(\%)\end{array}$ & $\begin{array}{c}\text { Theileria case } \\
\text { fatality risk } \$(\%)\end{array}$ & $\begin{array}{c}\text { Degree of tick } \\
\text { challenge } \S(\%)\end{array}$ & Age group & Epidemiologic state \\
\hline Central Equatoria & 52.20 & 56.00 & 71.01 & 31.61 & All & Unstable \\
\hline Eastern Equatoria & 24.50 & 35.33 & 65.03 & 32.15 & All & Unstable \\
\hline Western Equatoria & 25.00 & 42.03 & 59.33 & - & All & Unstable \\
\hline Jonglei & 29.50 & 30.00 & 75.10 & 14.17 & All & Unstable \\
\hline Lakes & 5.90 & 36.67 & 57.87 & 22.10 & All & Unstable \\
\hline
\end{tabular}

$\dagger$, Sero-prevalence computed as the number of calves sero-converted or total number of calves sampled.

$\$$, Derived from consultative discussions with the state veterinarians.

$\S$, Proportion of calf observations when ticks were present, based on adult tick counts.

TABLE 6: Criteria used to characterise endemic stability to Anaplasma marginale in the five states of South Sudan.

\begin{tabular}{|c|c|c|c|c|c|c|}
\hline States & $\begin{array}{c}\text { Anaplasma marginale } \\
\text { sero-prevalence }(\%)\end{array}$ & $\begin{array}{c}\text { Anaplasma } \\
\text { morbidity (\%) }\end{array}$ & $\begin{array}{l}\text { Anaplasma case } \\
\text { fatality risk (\%) }\end{array}$ & $\begin{array}{l}\text { Degree of tick } \\
\text { challenge }(\%)\end{array}$ & Age group & Epidemiologic state \\
\hline Central Equatoria & 51.60 & 60.00 & 70.00 & 31.61 & All & Unstable \\
\hline Eastern Equatoria & 51.50 & 66.00 & 70.00 & 32.15 & All & Unstable \\
\hline Western Equatoria & 18.80 & 40.00 & 82.00 & No data collected & All & Unstable \\
\hline Jonglei & 62.50 & 48.00 & 70.00 & 14.17 & All & Unstable \\
\hline Lakes & 67.50 & 71.00 & 70.00 & 22.10 & All & Unstable \\
\hline
\end{tabular}

two bands at approximately $220 \mathrm{bp}$ and $280 \mathrm{bp}$. The second genotype had a lower band at $150 \mathrm{bp}$, and the higher was similar to T. parva Muguga at $280 \mathrm{bp}$. The final genotype had a band similar to T. parva Muguga at $280 \mathrm{bp}$ and a higher band at about $400 \mathrm{bp}$.

\section{Survey of herdsmen}

Interviews with the herdsmen revealed that they were not willing to provide information about their animals. However, the following direct observations were made. The study states are suitable for many types of agricultural activities, including livestock keeping. The lush pastures, huge trees and other types of vegetation indicate abundant rainfall. This was confirmed through the consultative discussions with the state veterinarians and herdsmen who indicated that only 3-4 months of the year are dry. This also means that many parts of the country are suitable habitat for the ticks and their respective diseases. Nearly all the herds observed were zebu breeds and the animals were generally in good health. Animals graze communally in designated areas and only separated in the evenings into herds belonging to different families. Members of the same family keep their animals together at night for ease of guarding them. It was of great epidemiological interest to note that about $90 \%$ of the herdsmen met, and even some of the livestock field personnel, did not associate ECF with ticks. Instead, they viewed ticks as a major problem only because of their direct physical effects on the animals. This was considered an instrumental factor in the planning of future tick and tickborne diseases control interventions. 
Despite the general lack of information on TBD, however, there are some indications that livestock keepers are aware of the existence of ECF and other TBD (the majority of herdsmen described clinical signs compatible with those of ECF in their calves), and they have perfected traditional methods of treating the diseases. A standard method of treatment observed during the field investigation, is the burning of lymph nodes with a hot iron and the cauterisation of them with caustic juice derived from a plant. Farmers reported that this treatment is effective when applied early in the course of the disease. However, it was difficult to substantiate whether the recovery is an outcome of the treatment or because of an inborn resistance of the calves to the diseases. Indigenous cattle are known to develop resistance to the vector tick and to tick-borne diseases (Norval et al. 1992). The fact that the case fatality rate from ECF was reported to be so high (Table 5), puts into question the herdsmen's interpretation of the effectiveness of the treatment.

Currently, control of tick-borne diseases is minimal and limited to irregular use of acaricides (mainly to control tick infestation; herdsmen reportedly used acaricides when sufficient funds became available) and chemotherapy. The herdsmen also control ticks by the application of cattle urine mixed with ash. The main constraints to proper control were found to be a lack of infrastructure (service providers, dipping facilities and drug outlets). There was also a general lack of information on livestock husbandry, for instance, the failure of herdsmen to associate ticks with ECF. Generally there is very limited infrastructure in South Sudan to support large scale livestock interventions. There are, for example, only two dip tanks (one in Central Equatoria, and one in Western bahr Elghazal State) and about eight (Central Equatoria [2], Eastern Equatoria [2], Unit [2] and Warrap Sate [2]) functional checkpoints and one quarantine facility in Eastern Equatoria, in the country. Apart from the active network of community animal health workers, there are very few skilled personnel, and communication and input markets are also lacking. The majority of the herdsmen reported a lack of input and extension services as constraints to livestock keeping.

\section{Discussion}

The objective of this study was to investigate epidemiological parameters that may influence the development of tick and tick-borne disease control strategy for South Sudan. The study areas were purposefully selected based on the perceived ticks and tick-borne disease risk. In pastoral production systems, calves and other young animals are usually left to graze near the homestead; hence they were considered to be resident and the survey findings are therefore a true picture of the existing tick and tick-borne disease situation in the given area. Results obtained by Deem et al. (1993) suggest that, when sero-prevalence results from cross-sectional studies are used to determine the epidemiological status of tick-borne diseases in a given production system, it is important that sero-prevalence is assessed in homogenous systems if they are to be interpreted. Such homogenous systems should incorporate the same cattle type (in terms
TABLE 7: Band sizes (bp) of different Polymerase Chain Reaction deoxyribonucleic acid from samples examined for p104 allele.

\begin{tabular}{|c|c|c|}
\hline Sample & Bands size (bp) & Geneotype similarity \\
\hline EEM 25 & $220-280$ & Theileria parva Muguga \\
\hline EET 65 & $280-400$ & Theileria parva Kiambu 5 \\
\hline EEKN 69 & $280-400$ & Theileria parva Kiambu 5 \\
\hline EEM 44 & $150-280$ & - \\
\hline EET 54 & $280-400$ & Theileria parva Kiambu 5 \\
\hline EEM 29 & $280-400$ & Theileria parva Kiambu 5 \\
\hline EEM 31 & $150-280$ & Unknown \\
\hline EEM 24 & $220-280$ & Theileria parva Muguga \\
\hline EET 27 & $280-400$ & Theileria parva Kiambu 5 \\
\hline EEM 47 & $280-400$ & Theileria parva Kiambu 5 \\
\hline EET 39 & $280-400$ & Theileria parva Kiambu 5 \\
\hline EET 63 & $280-400$ & Theileria parva Kiambu 5 \\
\hline EET 51 & $150-280$ & - \\
\hline EET 30 & $280-400$ & Theileria parva Kiambu 5 \\
\hline EET 57 & $150-280$ & - \\
\hline JGB 88 & $220-280$ & Theileria parva Muguga \\
\hline LKA 29 & $280-400$ & Theileria parva Kiambu 5 \\
\hline LKA 22 & $280-400$ & Theileria parva Kiambu 5 \\
\hline LKA 21 & $280-400$ & Theileria parva Kiambu 5 \\
\hline$+\mathrm{F}$ & $220-280$ & Theileria parva Muguga \\
\hline CEL 3 & $220-280$ & Theileria parva Muguga \\
\hline JGB 97 & $220-280$ & Theileria parva Muguga \\
\hline JGB 30 & $220-280$ & Theileria parva Muguga \\
\hline JGB 45 & $220-280$ & Theileria parva Muguga \\
\hline JGB 41 & $220-280$ & Theileria parva Muguga \\
\hline JGB 92 & $280-400$ & Theileria parva Kiambu 5 \\
\hline CEL 48 & $220-280$ & Theileria parva Muguga \\
\hline CET 51 & $220-280$ & Theileria parva Muguga \\
\hline CET 46 & $280-400$ & Theileria parva Kiambu 5 \\
\hline CET 48 & $220-280$ & Theileria parva Muguga \\
\hline CEJ 17 & $220-280$ & Theileria parva Muguga \\
\hline CEJ 9 & $220-280$ & Theileria parva Muguga \\
\hline CEJ 21 & $280-400$ & Theileria parva Kiambu 5 \\
\hline CET 53 & $280-400$ & Theileria parva Kiambu 5 \\
\hline CET 35 & $280-400$ & Theileria parva Kiambu 5 \\
\hline CET 44 & $280-400$ & Theileria parva Kiambu 5 \\
\hline LEK 29 & $280-400$ & Theileria parva Kiambu 5 \\
\hline CEK 40 & $280-400$ & Theileria parva Kiambu 5 \\
\hline CET 54 & $280-400$ & Theileria parva Kiambu 5 \\
\hline CEJ 32 & $280-400$ & Theileria parva Kiambu 5 \\
\hline CET 22 & $220-280$ & Theileria parva Muguga \\
\hline CET 45 & $220-280$ & Theileria parva Muguga \\
\hline
\end{tabular}

of their susceptibility), the appropriate age-group, sampled at the appropriate time of year and within appropriate strata of the ecological cline. However, this requirement is more important if herd sizes are very small, as in the zerograzing smallholder herds; in this study larger pastoral herds were used, and given the communal grazing and husbandry practices whereby, at one point in time, almost the entire national herd congregate along the Nile River during the dry spell. From an epidemiological perspective, the study population was therefore assumed to be a single, homogenous, free mixing host population.

It was not possible to establish the ECF specific case fatality risk reliably. However, consultative discussions with the state veterinarians and the producers in the study areas indicate that case fatality risk caused by ECF as high as $80 \%-100 \%$ 
occurs, depending on the time of the year. These estimates compared very well with the results of a recent unpublished socio-economic study in the same areas, but the estimates are very high compared to what would be expected in the East Africa region where calf mortality ascribable to tick-borne diseases range between $30 \%$ and $50 \%$, to as low as $2 \%-3 \%$ (Norval et al. 1992), depending on the different tick-control practices and the existing endemic situation. However, ECF specific case fatality risk ranging between $50 \%$ and $100 \%$ are not uncommon in the region. Cases of death are common especially in high-susceptible exotic breeds, such as Bos taurus, and their crosses with indigenous breeds, but the indigenous breeds are also at risk, particularly in endemically unstable or epidemic areas (Norval et al. 1992).

Data suggest that the majority of the calves are born with very little or no (the negative sign) maternal antibodies to the major tick-borne parasites studied (Figure 3), but they sero-convert by the age of 3 months, the age at which, according to the questionnaire results, they go out for grazing. However, the acquired antibodies quickly decay with advancing age; in such a way that the majority of the calves have less than $15 \%$ sero-prevalence at 11 months. This could be ascribed to several reasons. Firstly, the majority of dams are sero-negative to the common tick-borne diseases. Secondly, there may be a low tick challenge and if the tick challenge is good, then it is likely that several factors such as the number, viability, and infectivity of sporozoites within the salivary glands, pathogen and T. parva strain differences, and the duration of tick feeding could interplay to influence the ability of the $R$. appendiculatus to acquire T. parva and the transmission of T. parva from infected ticks to cattle. In endemic areas the majority of infected ticks likely to become infected by feeding on carrier and clinically affected cattle (Medley, Perry \& Young 1993), but the findings recorded (Table 1) and the presence of schizonts in only $3.11 \%$ of the study population suggest that the subsequent instars would then possibly inoculate relatively fewer parasites, reducing the overall tick-infectivity level. This is probably corroborated by the recent findings by Ogden et al. (2003) that the level of parasitaemia could affect the rate of acquisition of T. parva by ticks and the subsequent cattle challenge. However, it is worth noting that, in a given cattle production system, the level of infection challenge will depend on classical risk factors such as climatic suitability for ticks, grazing practices and range (and thus mixing with other potentially infected cattle), tick-control practices and host susceptibility. Thirdly, herdsmen in the study areas use cattle urine mixed with ash (the ammonia in urine suffocates the ticks, whilst ash is used as a binding material) to remove ticks from animals. This practice interrupts tick challenge and may also contribute to the observations recorded (see Figure 3). Fourthly, the relatively high risk levels, particularly of anaplasmosis, could result in early and high calf mortality which could also influence the resulting sero-prevalence patterns in the population, as calves do not live long enough to develop immunity.
The observed high level of tick infection (Table 3) is probably because of the fulminating theileriosis, and indeed the ticks with high infection prevalence in Jonglei State came from a cattle camp that reportedly had an outbreak of ECF just before the survey.

The high prevalence of tick infection is far above the recorded levels of $2.6 \%$ and $2.3 \%-14.2 \%$ in more endemic areas of Tanzania and Kenya (Ogden et al. 2003; Oura et al. 2004).

Variations in sero-prevalence in the study states suggest that tick-borne diseases exist in different epidemiological states, so that morbidity and mortality may fluctuate from season to season and from herd to herd. This study, as in other previous studies in the East African region (Gitau et al. 1997; RubaireAkiiki et al. 2004, 2006), demonstrated the crucial influence of farm circumstances, grazing systems and agro-ecological zones on the variation in tick and tick-borne diseases risk, both spatially and temporally. Thus, herdsmen and the veterinarians in South Sudan need to pay close attention to varying ECF challenge in making decisions on ECF and other TBD control.

The overall sero-prevalence of $27.30 \%$ and $31.30 \%$ for T. parva and T. mutans respectively, suggest that endemic instability exists in the study population and an outsized proportion of the population is likely to succumb to clinical theileriosis. On the other hand the overall sero-prevalence of $57.60 \%$ and $52.80 \%$ for $A$. marginale and B. bigemina is suggestive of a state of medium endemic stability. It has been suggested that high sero-conversion risks are associated with little or no clinical cases of tick-borne diseases (Norval et al. 1992). However, the high reports of clinical cases (Tables 5 and 6), indicate that this may not be the case, and clearly sero-prevalence alone is not a reliable indicator of endemic stability.

The results of this study have important implications for future ECF and other tick-borne disease control in South Sudan. In our opinion, herdsmen and veterinarians in South Sudan should aim for managing tick populations and tickborne diseases within economically acceptable limits, in which the risks of tick-borne diseases outbreak are minimal; this can be achieved by ensuring that cattle are immune. Immunity may be maintained by simply ensuring that tick numbers are high enough to perpetuate endemic stability. Any control programme should therefore aim to provide sufficient tick control to increase cattle productivity, whilst not reducing tick numbers to the extent that endemic stability is adversely affected. Where endemic stability is not possible (as a result of factors affecting transmission of parasites from either clinical cases or carrier animals) vaccination may be required as a disease control measure.

The low and intermediate sero-conversion risks observed (Table 5) in the study states suggest that ECF immunisation of the study population is justified. Fortunately, the similarity (Table 7) between the Southern Sudan and the Muguga T. parva isolates implies that the Muguga cocktail vaccine 
can be used in South Sudan. With immunisation there will be a greater likelihood of the development of endemic stability; wider immunisation coverage combined with modified acaricide control strategies to allow sufficient tick challenge probably offers the best prospect for establishing and maintaining endemic stability in the Southern Sudan cattle population. The relatively high sero-prevalence of B. bigemina (Table 4) and A. marginale (Table 6) suggests that a reduction in the frequency of acaricide application following ECF immunisation in the study states would therefore not be expected to result in an increased incidence of these diseases.

It is quite clear from this study that attention will have to be paid to variations in tick and TBD risk, both spatially (tick and TBD dynamics change over relatively short geographical distances) and temporally (seasonally and secularly), to develop optimal combinations of control strategies for TBD under various husbandry and ecological conditions. In the short term, any control strategy to be adopted should be supported by strategically located quarantine or holding grounds with dipping facilities, and enacting and enforcing appropriate legislations that promote good husbandry practices by the herdsmen. It was of epidemiological interest to note that about $90 \%$ of the herdsmen and community animal health workers could not associate the presence of ticks with the occurrence of TBD; therefore, for a successful control strategy, an element of public awareness through extension services should feature strongly, and such a strategy should be supported in addition by a strong epidemiological surveillance and laboratory confirmation of the suspected cases.

\section{Conclusion}

The purpose of this study was to investigate epidemiological parameters of tick-borne diseases in five states of South Sudan, and their uses in future control strategies. The results contribute to the epidemiological understanding that bovine theileriosis, anaplasmosis and babesiosis are endemic and exist in an unstable state. The T. parva isolates from South Sudan matched very well with T. parva Kiambu and T. parva Muguga, and consequently the Muguga cocktail can be used for immunisation of cattle in South Sudan.

\section{Acknowledgements}

We are grateful to the herdsmen who gave their time and allowed us to sample their animals. The help and participation of staff in the Department of Veterinary Services of the Ministry of Animal Resources and Fisheries of the Government of South Sudan, is highly appreciated. This survey was funded by the Livestock Epidemio-surveillance Project Southern Sub-Project coordinated by Vétérinaires Sans Frontières, Belgium.

\section{Competing interests}

None of the authors of this paper has a financial or personal relationship with other people or organisations that could inappropriately influence or bias the content of the paper.

\section{Authors' contributions}

F.M.K. (Ministry of Livestock and Fisheries Development) was the project leader and was responsible for the study design and data analysis. G.K.M. (Sokoine University of Agriculture) and P.F.M. (Veterinary Investigation Centre, Arusha) carried out the laboratory work, and A.M.K. (Central Veterinary Laboratory, Dar es Salaam) and R.J.W. (Ministry of Animal Resources and Fisheries, South Sudan) were responsible for the field work.

\section{References}

Bazarusanga, T., Vercruysse, J., Marcotty, T. \& Geysen, D., 2007, 'Epidemiological studies on Theileriosis and the dynamics of Theileria parva infections in Rwanda', Veterinary Parasitology 143, 214-221. http://dx.doi.org/10.1016/j. vetpar.2006.09.009, PMid:17055650

Bishop, R., Geysen, D., Spooner, P., Skilton, R., Nene, V., Dolan, T. et al., 2001, 'Molecular and immunological characterisation of Theileria parva stocks which are components of the "Muguga cocktail" used for vaccination against East Coast fever in cattle', Veterinary Parasitology 94, 227-2377. http://dx.doi.org/10.1016/ S0304-4017(00)00404-0

Buscher, G. \& Otim, B., 1986, 'Quantitative studies on Theileria parva in the salivary glands of Rhipicephalus appendiculatus adults: Quantitation and prediction of infection', International Journal of Parasitology 16, 93-100. http://dx.doi. org/10.1016/0020-7519(86)90071-8

De Vos, A.J., 1992, 'Distribution, economic importance and control measures for Babesia and Anaplasma', in T.T. Dolan (ed.), Recent developments in the control of anaplasmosis, babesiosis and cowdriosis, pp. 3-12, ILRAD, Nairobi.

Deem, S.L., Perry, B.S., Katende, J.M., McDermott, J.J., Mahan, S.M., Maloo, S.H. et al., 1993, 'Variations in prevalence rates of tick-borne disease in Zebu cattle by agro-ecological zone: Implications for East Coast fever immunization', Preventive Veterinary Medicine 6, 171-187. http://dx.doi.org/10.1016/0167 5877(93)90064-Z

Gitau, G.K., Perry, B.D., Katende, J.M., McDermott, J. Jorzaria, S.P. \& Young, A.S 1997, 'The prevalence of serum antibodies to tick-borne infections in cattle in smallholder dairy farms in Murang'a District, Kenya: A cross-sectional study', smallholder dairy farms in Murang'a District, Kenya: A cross-sectional study',
Preventive Veterinary Medicine 30, 95-107. http://dx.doi.org/10.1016/S0167Preventive Veterinar
5877(96)01100-2

Hoogstraal, H., 1956, 'Ticks of the Sudan', United States Naval Medical Research Unit No. 3. Cairo, Egypt, Research report NM 005 050.29.07.

Julla, I.I., 1985, 'Theileriosis in Southern Sudan', in A.D. Irvin (ed.), Immunization against theileriosis in Africa: Proceedings of a joint workshop, ILRAD, Nairobi, October 01-05, 1984, pp. 27-30.

Julla, I.I., 1993, 'Progress in research on theileriois in Equatoria State, Sudan', in T.T.Dolan (ed.), Ticks and tick-borne disease control: Proceedings of a joint OAU and ILRAD workshop, Kampala, September 12-14, 1991, p. 8.

Julla, I.I., Tingwa, N.O. \& Kwajok, V.L., 1989, 'Theileriosis in the Equatorial region of Sudan', in T.T Dolan (ed.), Theileriosis in Eastern, Central and Southern Africa: Proceedings of a workshop on East Coast fever immunisation, Lilongwe, September 20-22, 1988, pp. 19-21.

Katende, J.M., Gooddeeris, B.M., Morzaria, S.P., Nkonge, C.G. \& Musoke, A.J., 1990 'Identification of a Theileria mutans-specific antigen for use in an antibody and antigen detection ELISA', Parasite Immunology 12, 419-433. http://dx.doi. org/10.1111/j.1365-3024.1990.tb00978.x, PMid:1698274

Katende, J.M., Toye, P., Skilton, R.A., Nene, V., Morzaria, S.P. \& Musoke, A.J., 1998 , 'An ELISA for detection of Theileria parva antibodies in cattle using a recombinant polymorphic immunodominant molecule', Parasitology Research 84, 408-416. http://dx.doi.org/10.1007/s004360050419, PMid:9610640

Kivaria, F.M., 2006, 'Estimated direct economic costs associated with tick-borne diseases on cattle in Tanzania', Tropical Animal Health and Production 38, 291299. http://dx.doi.org/10.1007/s11250-006-4181-2, PMid:17137131

Kivaria, F.M., 2007, 'The control of East Coast Fever in Africa: a constant battle for impoverished dairy farmers', The Veterinary Journal 174, 221-222. http://dx.doi. org/10.1016/j.tvjl.2006.09.001, PMid:17092747

Mahoney, D.F., 1977, 'Babesia of Domestic Animals', in J.P. Kreier (ed.), Parasitic protozoa, vol. IV, pp. I-14, Academic Press, New York.

Maloo, S.H., Thorpe, W., Kioo, G., Ngumi, P., Rowlands, G.J. \& Perry, B.D., 2001, 'Seroprevalences of vector-transmitted infections of smallholder dairy cattle in coastal Kenya', Preventive Veterinary Medicine 52, 1-16.

Marufu, M.C., Chimonyo, M., Dzama, K. \& Mapiye, C., 2010, 'Seroprevalence of tickborne diseases in communal cattle reared on sweet and sour rangelands in a semi-arid area of South Africa', The Veterinary Journal 84, 71-76. http://dx.doi. org/10.1016/j.tvjl.2009.02.014, PMid:19733490

Medley, G.F., Perry, B.D. \& Young, A.S., 1993, 'Preliminary analysis of the transmission dynamics of Theileria parva in eastern Africa', Parasitology 106, 25I-264.

Norval, R.A.I., Perry, B.D. \& Young, A.S. (eds.), 1992, The epidemiology of theileriosis in Africa, London, Academic press.

Ogden, N.H., Gwakisa, P., Swai, E., French, N.P., Fitzpatrick, J., Kambarage, D. et al., 2003, 'Evaluation of PCR to detect Theileria parva in field collected tick and bovine samples in Tanzania', Veterinary Parasitology 112, 117-183. http://dx.doi. org/10.1016/S0304-4017(02)00448-X 
Oura, C.A., Bishop, R.P., Wampande, E.M., Lubega, G.W. \& Tait, A., 2004, 'Application of a reverse line blot assay to the study of haemoparasites in cattle in Uganda', ijpara.2003.12.012, PMid:15064125

Perry, B.D. \& Young, A.S., 1995, 'The past and future roles of epidemiology and economics in the control of tick-borne diseases of livestock in Africa: The case of theileriosis', Preventive Veterinary Medicine 25, 107-120. http://dx.doi. org/10.1016/0167-5877(95)00546-3

Perry, B.D., Deem, S.L., Medley, G.F., Morzaria, S.P. \& Young, A.S., 1992, 'The ecology of Theileria parva infections of cattle and the development of endemic stability' in U.G. Munderloh \& T.J. Kurtti (eds.), Proceedings of the 1st international conference on tick-borne pathogens at the host-vector interface: an agenda for research, September 15-18, 1992, pp. 1-7.

Rubaire-Akiiki, C.M., Okello-Onen, J., Musunga, D., Kabagambe, E.D., Vaarst, M., Okello, D. et al., 2006, 'Effect of agro-ecological zone and grazing system on incidence of East coast fever in calves in Mbale and Sironko Districts of Eastern Uganda', Preventive Veterinary Medicine 75, 251-266. http://dx.doi org/10.1016/j.prevetmed.2006.04.015, PMid:16797092
Rubaire-Akiiki, C., Okello-Onen, J., Nasinyama, G.W., Vaarst, M., Kabagambe, E.K., Mwayi, W. et al., 2004, 'The prevalence of serum antibodies to tick-borne infections in Mbale District, Uganda: The effect of agro-ecological zone, grazing management and age of cattle', Journal of Insect Science 4, viewed n.d., from http://insectscience.org/4.8/

Swai, E.S., French, N.P., Karimuribo, E.D., Fitzpatrick, J.L., Bryant, M.J., Brown, P.E. et al., 2005, 'Spatial and management factors associated with exposure of smallholder dairy cattle in Tanzania to tick-borne pathogens', International Journa of Parasitology 35, 1085-1096. http://dx.doi.org/10.1016/j.ijpara.2005.04.006, Mid:16023121

Walker, A.R., Bouattour, A., Camicas, J.-L., Estrada-Pena, A., Horak, I.G., Latif, A.A. et al., 2003, Ticks of Domestic Animals in Africa, Bioscience reports, The University of Edinburgh, Scotland, U.K.

Wright, P.F., Nilsson, F., Van Rooij, E.M.A., Lelenta, M. \& Jeggo, M.H., 1993 'Standardisation and validation of enzyme-linked immuno-sorbent assay techniques for the detection of antibody in infectious disease diagnosis', Scientific and Technical Review (OIE) 12, 435-450. PMid:8400384 